significant overlap between their attenuation ranges, most crucially between necrotic core and fibrous plaque. Recently introduced dual energy CT (DECT) permits acquisition of 2 different energy data sets simultaneously, with the change in attenuation of plaque components to different energies depending upon their material composition. We therefore examined whether DECT was better than single energy CT in determining plaque components defined by virtual histology IVUS.

Methods 20 patients underwent DECT and 3-vessel VH-IVUS. CT data was obtained at peak voltages of $100 \mathrm{kV}$ and $140 \mathrm{kV}$. 52 plaques were chosen with either homogenous fibrous plaque or confluent areas of calcified plaque or necrotic core as defined by VH-IVUS. VHIVUS images were co-registered and orientated with the corresponding CT images using distance from coronary ostia and fiduciary markers (Abstract 113 figure 1). Multiple regions of interest (ROI) were placed within the plaque components or in lumen on cross sectional CT images pre-classified by VH-IVUS (Abstract 113 figure 1). ROI densities were measured (in Hounsfield Units) and assigned to the plaque component. A dual energy index (DEI) was created for each component, defined as the ratio of the difference in attenuation at 2 different energies / sum of attenuation with 1000 added to each attenuation value to avoid negatives.

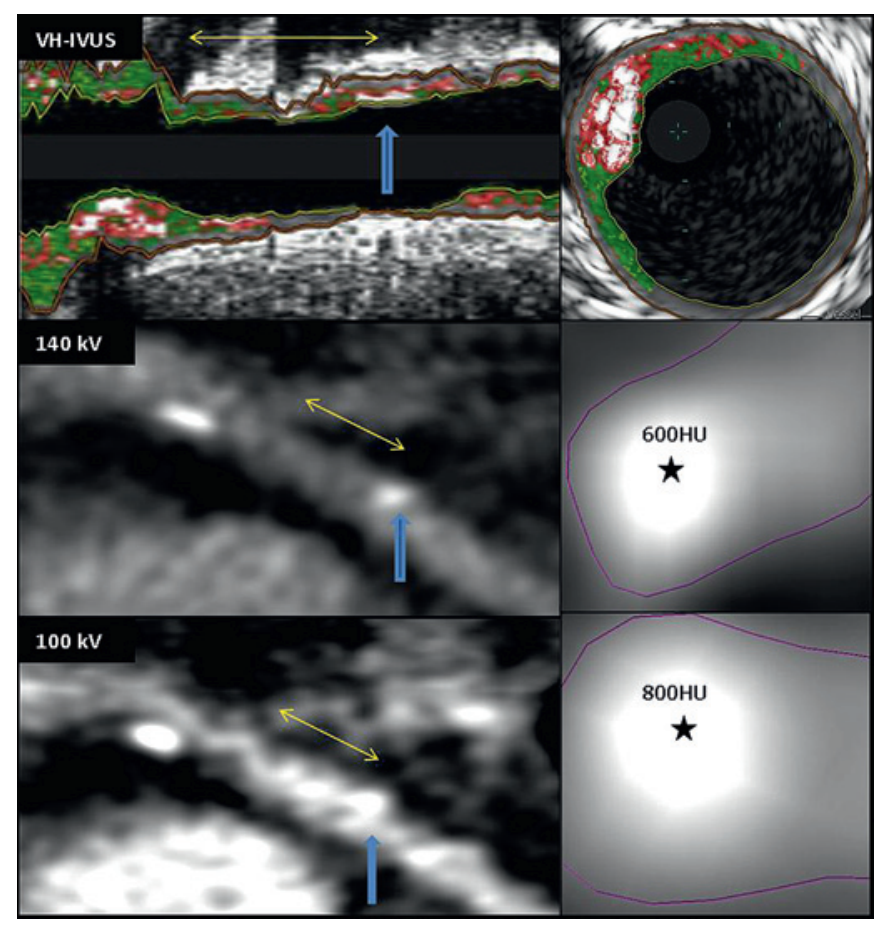

Abstract 113 Figure 1 Demonstration of plaque co-registration between VH-IUS and 140kV/100kV CT data sets. Calcified plaque is identified $5 \mathrm{~mm}$ from side branch adjacent to characteristic calcification (yellow line). Cross section taken through this plaque (blue arrow) and following orientation with VH-IVUS cross section $\mathrm{HU}$ region of interest sampling is performed in calcified plaque.

Results Attenuation values for 1088 ROIs were measured from 70 paired data sets at $100 \mathrm{kV}$ and $140 \mathrm{kV}$ creating 70 DEIs (12 necrotic core, 11 fibrous plaque, 29 calcified plaques and 18 lumen). Values obtained using a single energy data set showed good differentiation between calcified plaque and all others $(\mathrm{p}<0.05)$, but considerable overlap between necrotic core and fibrous plaque $(\mathrm{p}=\mathrm{ns})$ (Abstract 113 figure 2A) (Abstract 113 table 1). In DECT, lumen (iodinated contrast) showed the greatest change in attenuation and hence had the highest DEI. Necrotic core had the lowest DEI and could be distinguished from all other components $(p<000.1)$ Importantly, in contrast to the single energy data, DEI derived from both energy data sets permitted resolution of necrotic core and fibrous plaque without overlap (Abstract 113 figure 2B).
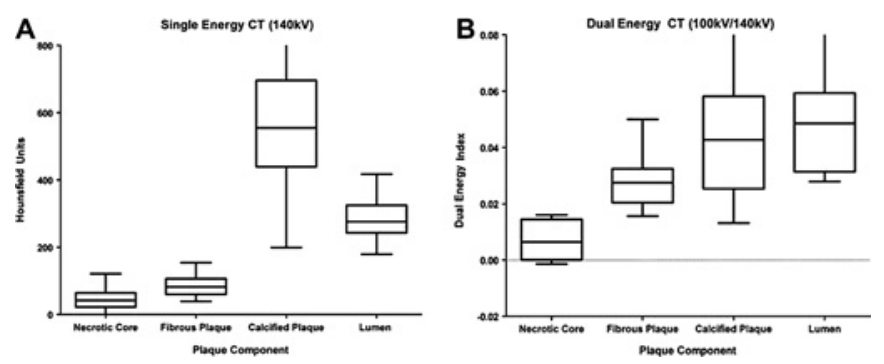

Abstract 113 Figure 2 (A) Defined CT attenuation spectra of plaque components using a single energy $(140 \mathrm{kV})$, calcified plaque is distinguishable from all others but necrotic core and fibrous plaque overlap. (B) The use of dual energy index from the attenuation data at 2 energies $(100 / 140 \mathrm{kV})$ allows significant separation of necrotic core and fibrous plaque $(p<0.05)$ (Tukeys multiple comparison test).

Abstract 113 Table 1

\begin{tabular}{lrrll}
\hline $\begin{array}{l}\text { Plaque } \\
\text { Component }\end{array}$ & $\begin{array}{l}\text { 100 kV mean } \\
\text { HU (SD) }\end{array}$ & $\begin{array}{l}\text { 140 kV mean } \\
\text { HU (SD) }\end{array}$ & $\begin{array}{l}\text { Mean Difference } \\
(\mathbf{1 0 0}-\mathbf{1 4 0} \mathbf{~ k V})\end{array}$ & $\begin{array}{l}\text { Dual Energy } \\
\text { Index (mean) }\end{array}$ \\
\hline Necrotic Core & $57.26(42.20)$ & $42.69(31.51)$ & 14.57 & 0.0071 \\
Fibrous Plaque & $148.30(49.47)$ & $84.60(30.34)$ & 63.69 & 0.0283 \\
Calcified Plaque & $733.10(226.7)$ & $582.20(194.9)$ & 150.9 & 0.0450 \\
Lumen & $411.5(82.27)$ & $282.90(55.93)$ & 128.6 & 0.0483 \\
\hline
\end{tabular}

Conclusions The additional attenuation data provided by DECT improves the differentiation of plaque components when compared to conventional single energy CT. In particular, DECT may allow better differentiation of necrotic core and fibrous plaque, a weakness of conventional cardiac CT, allowing for more accurate non-invasive identification of vulnerable plaques.

\section{RADIATION DOSES TRENDS FROM CARDIAC CT USING A CARDIAC SPECIFIC CONVERSION FACTOR: SYSTEM UNDERSTANDING \& AN OPTIMISATION STRATEGY SIGNIFICANTLY REDUCES THE DOSE TO THE PATIENTS IN A CLINICAL SERVICE}

doi:10.1136/heartjnl-2011-300198.114

${ }^{1} \mathrm{O}$ E Gosling, ${ }^{1} \mathrm{~S}$ lyengar, ${ }^{1} \mathrm{R}$ Loader, ${ }^{1} \mathrm{G}$ Morgan-Hughes, ${ }^{2} \mathrm{~W} D$ Strain, ${ }^{3} \mathrm{C}$ Roobottom. ${ }^{1}$ Plymouth Hospitals NHS Trust, Plymouth, UK; ${ }^{2}$ Peninsula College of Medicine and Dentistry, Exeter, UK; ${ }^{3}$ Peninsula College of Medicine and Dentistry, Plymouth, UK

Background CT coronary angiography CTCA now has an established role in the investigation of patients with chest pain. Under the IRMER regulations radiation doses to patients should be kept as low as reasonably practical (ALARP). Previous publications have used a chest conversion factor to calculate the effective dose ( $\mathrm{mSv})$ from CTCA. We have previously demonstrated that chest conversion factors significantly under-estimate the effective dose to the patient when applied to CTCA and have calculated a cardiac specific conversion factor of $0.028 \mathrm{mSv}$ (mGy.cm)-1. Our department follows the ALARP ethos and has implemented new technologies together with physician training to reduce the radiation dose from CTCA. We aimed to investigate what impact the implementation of new technologies has had on the radiation dose of CTCA.

Method All patients who were coded as attending for a cardiac CT scan on the PACS and CRIS systems were included in the analysis Scan indication included: rule out coronary artery disease, CABG assessment, pre-EP studies and problem solving. CT scanning 
between September 2007 and August 2010 was included; the total dose for the whole examination is used including the scout and nonenhanced scan (calcium score). Scans were performed on a Lightspeed VCT or HD750 (GE Healthcare). To calculate the effective dose a conversion factor was applied to the dose length product of each examination. The DLP is the radiation dose in one CT slice multiplied by the length of the scan. A cardiac specific conversion factor was used rather than a chest conversion factor (0.014) which significantly underestimates the effective dose from CTCA. Data was transformed and expressed as a geometric mean with $99 \%$ CI. For each analysis period all scans were included; retrospective, prospective, low $\mathrm{kV}$ and zero padding.

Results In the 3-year period 1736 scans were performed. The mean radiation dose in the first 6 months of the study (retrospective gating) was $29.6 \mathrm{mSv}$; using the accepted conversion factor at the time the mean dose was $14.9 \mathrm{mSv}$. In March 2008 prospective ECG gating was installed; this resulted in a halving of the mean radiation dose to $13.6 \mathrm{mSv}$. In March 2009 the scanner parameters was set to zero padding and $100 \mathrm{KV}$ reducing the dose to $7.4 \mathrm{mSv}$. For the final 6 months the mean radiation dose for a cardiac scan was $5.9 \mathrm{mSv}$; this Abstract 114 figure 1 incorporates scans performed with standard filtered back projection, iterative reconstruction, high definition scanning and retrospective ECG gating for a variety of differing clinical scenarios.

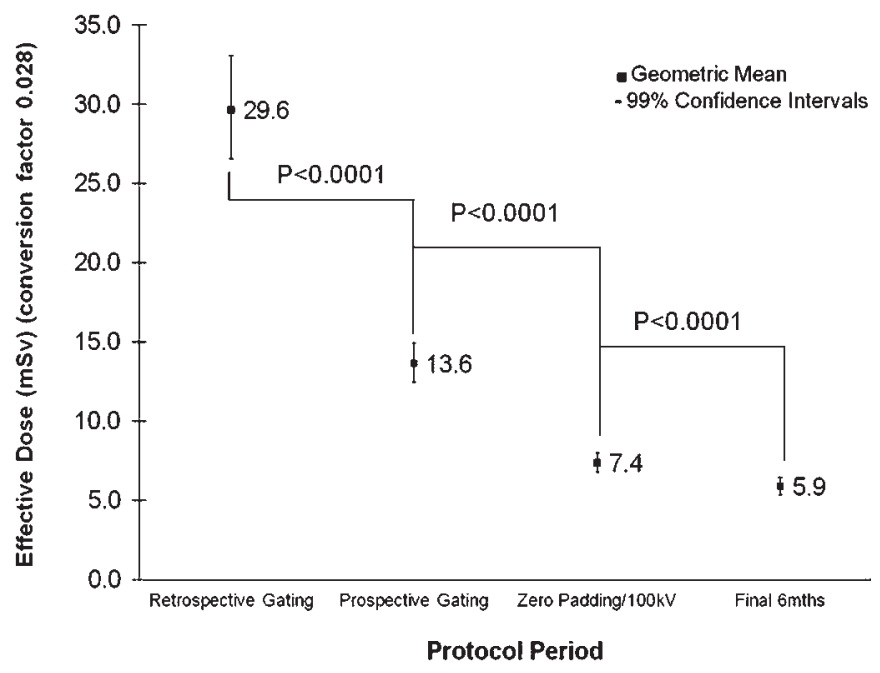

Abstract 114 Figure 1 Effective dose (mSv) by protocol period.

Conclusion The introduction of dose saving strategies and appropriate physician training has lead to a significant reduction in the radiation dose from cardiac CT. As CTCA programmes become established in hospitals around the UK it is important that clinicians have the appropriate training and experience to keep the radiation dose to the patients as low as reasonably practical.

Abstract 114 Table 1

\begin{tabular}{llllc}
\hline & $\begin{array}{l}\text { Retrospective } \\
\text { Gating-dose } \\
\text { modulation }\end{array}$ & $\begin{array}{l}\text { Prospective } \\
\text { gating }\end{array}$ & $\begin{array}{l}\text { Zero padding- } \\
\mathbf{1 0 0 ~} \mathbf{~ V}\end{array}$ & $\begin{array}{c}\text { Final 6 } \\
\text { months }\end{array}$ \\
\hline $\begin{array}{l}\text { Number of Patients } \\
\text { Mean Effective }\end{array}$ & 150 & 489 & 636 & 461 \\
$\begin{array}{l}\text { Dose (mSv) } \\
\text { Cls (99\%) (mSv) }\end{array}$ & 29.6 & 13.6 & 7.4 & 5.9 \\
& 26 & 14.9 & 8 & 6.5 \\
& 26.6 & 12.5 & 6.8 & 5.3 \\
\hline
\end{tabular}
115 ATRIAL HIGH RATE EPISODES AND ATRIAL FIBRILLATION
BURDEN: DO THEY HAVE SIMILAR ASSOCIATION WITH CARDIAC REMODELLING?

doi:10.1136/heartjnl-2011-300198.115

C W Khoo, S Krishnamoorthy, G Dwivedi, B Balakrishnan, H S Lim, G Y H Lip. University Department of Medicine Centre for Cardiovascular Sciences, City Hospital, Birmingham, UK

Background and Objectives Contemporary pacemaker devices allow quantification of atrial high-rate episodes (AHREs) and atrial fibrillation burden (AFB) accurately. Cumulative ventricular pacing (Vp) is associated with development of atrial fibrillation, but it is not clear if AHREs and AFB share similar pathophysiologic associations with left atrium (LA) and ventricle (LV) function and remodelling. Methods In total, 87 patients with dual-chamber pacemaker underwent two-dimension (2D) and tissue Doppler imaging (TDI) echocardiography. LA volume (LAV) was evaluated by area-length method and indexed to body surface area. Septal A' was used to measured regional LA function. LV systolic and diastolic parameters were evaluated by mitral inflow velocity (E, A, E/A), LV ejection fraction (biplane Simpson's) and septal TDI velocity. The presence of AHREs (defined by atrial-rate $\geq 220$ beats $/ \mathrm{min}$ and $\geq 5$ minutes) and $\mathrm{AFB}$ were derived from pacemaker diagnostics. Plasma markers of remodelling, matrix metalloproteinases-1 (MMP1) and tissue inhibitors of metalloproteinases-1 (TIMP1), were analysed by ELISA. Results Baseline characteristics and comorbidities were comparable between groups (Abstract 115 table 1). Patients with AHREs had significantly larger indexed LAV $(p=0.011)$ and higher cumulative $\mathrm{Vp}(\mathrm{p}=0.012)$, but this was not associated with elevation of MMP1 and TIMP1. Plasma markers, LV systolic and diastolic parameters were comparable between groups. In patients with AHREs, the AFB ranged from 0 to $99 \%$ and correlated with $\mathrm{E} / \mathrm{A}(\mathrm{r}=0.966, \mathrm{p}<0.001)$, and inversely correlated with late acceleration velocity (A) $(r=-0.612, p=0.009)$. On linear regression analysis, $A, E / A$, septal $A^{\prime}$ were independently associated with AFB (all $p<0.01$ ).

Conclusion Cumulative $\mathrm{Vp}$ and increased LAV are associated with the development of AHRE, but AFB is independently associated with changes in LA function and LV diastolic function. This study suggests AHREs and AFB have dissimilar pathophysiologic associations with left atrium and ventricle remodelling.

Abstract 115 Table 1

\begin{tabular}{lccl}
\hline & No AHRE $(\mathbf{n}=\mathbf{7 0})$ & AHRE $(\mathbf{n}=\mathbf{1 7})$ & $\mathbf{p ~ V a l u e ~}$ \\
\hline Age (years) & $71.0 \pm 11.6$ & $75.4 \pm 8.8$ & 0.1 \\
Body mass index $(\mathrm{kg} / \mathrm{m} 2)$ & $26.4 \pm 4.4$ & $27.6 \pm 4.7$ & 0.38 \\
Indexed LA volume $(\mathrm{ml} / \mathrm{m} 2)$ & $27.4 \pm 7.9$ & $34.8 \pm 9.4$ & 0.01 \\
LV ejection fraction (\%) & $52.8 \pm 11.9$ & $55.1 \pm 9.2$ & 0.4 \\
E/A & $0.8 \pm 0.2$ & $1.0 \pm 0.6$ & 0.23 \\
Septal A' (cm/s) & $8.9 \pm 2.2$ & $7.9 \pm 2.6$ & 0.16 \\
Septal S' (cm/s) & $6.6 \pm 1.8$ & $6.5 \pm 1.4$ & 0.71 \\
Septal E/E' & $13.7 \pm 6.2$ & $14.1 \pm 3.5$ & 0.74 \\
Percentage Vp & $21.9(1.8-99.0)$ & $98.6(41.0-99.9)$ & 0.01 \\
\hline
\end{tabular}

116 CRT OPTIMISATION: IMPROVING ECHOCARDIOGRAPHIC TECHNIQUES BY ACCOMMODATING BIOLOGICAL VARIABILITY WITHIN DIFFERENT ECHOCARDIOGRAPHIC PARAMETERS

doi:10.1136/heartjnl-2011-300198.116

P A Pabari, A Kyriacou, M Moraldo, B Unsworth, N Sutaria, J Mayet, A D Hughes, D P Francis. Imperial College London, London, UK

Background In optimisation of CRT (and even selection for implantation) we may have underestimated the impact of beat-to-beat 